\title{
Quality of life and Social Support among Children Living with HIV (CLHIV) in South India
}

\author{
Mrs. Bharathi ${ }^{1}$, Dr. Mamatha Shivananda Pai ${ }^{2}$, Dr. Baby S. Nayak ${ }^{3}$ \\ ${ }^{1} \mathrm{MSc}(\mathrm{N})$ graduate, Manipal College of Nursing, Manipal University, India \\ ${ }^{2,3}$ Professor, Department of Pediatric Nursing, Manipal College of Nursing, Manipal University,, India.
}

\begin{abstract}
This study was a descriptive correlational survey performed to find the quality of life and social support of children living with HIV (CLHIV). The subjects were 50 CLHIV selected using purposive sampling, living in one of the districts of South India attending ART plus centre of Distrcit hospital. Among 50 CLHIV, $23(46.1 \%)$ of the belonged to the age group of 16-18 years and $28(56 \%)$ subjects were males. Poor quality of life was found among 30(60\%) children and majority,43(86\%) of the subjects were found to have low social support. Statistically significant weak positive relationship $(r=.293 ; p=.039)$ between quality of life and social support of CLHIV. The present findings suggest that interventions to improve quality of life and social support could have a positive influence on CLHIV. An interventional study should be performed in future.
\end{abstract}

Keywords: CLHIV, social support, South India, quality of life.

\section{Introduction}

Infections like HIV and AIDS) are the most significant public health problem in developing countries .HIV develops very rapidly among infants and children. HIV infection in young children is especially fatal. The World Health Organization (WHO) reports HIV/AIDS as one of the world's killing infectious disease that claims more than 25 million lives in the past thirty years. Children living with HIV (CLHIV) are very prone to get opportunistic infections, because of their weak immune systems. Major causes of illness and death among infected infants are tuberculosis and pneumonia.

HIV-related stigma and discrimination continue to be a major obstacle to an effective HIV response in the world, it was found that the discrimination is still a problem among people living with HIV. [1]. Political Declaration on HIV/AIDS in the year 2011preapred a list of 10 targets to be met by 2015 . Among this $8^{\text {th }}$ point is eliminate HIV related stigma and discrimination.

In spite of programs and awareness, HIV-related stigma and discrimination continue to be present in all the areas, although the degree and severity vary. Stigma affects the social, educational and all other behaviour and lifestyle of individuals [2].

Discrimination against AIDS in Asia show that HIV-related stigma and discrimination were present in all the levels of society, with $80 \%$ of respondents reporting such experiences, including discrimination in the health sector $(54 \%)$, community $(31 \%)$, family $(18 \%)$ and the workplace $(18 \%)$. The project also found that women compared to men experience more HIV-related discrimination in their family and community [2].

Number of people living with HIV/AIDS (PLHIV) in the year 2011 in India was estimated to be 20.9 lakh. Children less than 15 years of age accounted for 7\% (1.45 lakh) of all infections and $86 \%$ were between 15-49 years of age. The estimated number of PLHIV in India reducing from 23.2 lakh in 2006 to 20.9 lakh in 2011. 53\% of prevalence was reported from four high prevalence States of South India (Andhra Pradesh, Karnataka, Maharashtra and TamilNadu). [3].

A case control study on quality of life was conducted by Mohit Gupta et al in 2013 on HIV infected children and other chronic ailments $(40+40)$ at the referral hospital in Northern India. A significantly improved quality of life of HIV infected children was observed among children with chronic illness than among children with HIV. The study concluded that the quality of life was better in HIV infected children than those suffering from chronic illness [4].

In a cross-sectional study conducted in Thailand measured quality of life among main caregivers of HIV-infected children. A total of 131 main caregivers answered the questionnaires which consisted of 5 main domains: general health, physical functioning, symptoms, psychological wellbeing, and social and role functioning. A significant correlation was found between health care utility and physical functioning, symptoms, and social and role functioning [5].

Impact of HIV/AIDS among children was studied in Yunnan in the year 2006. A Study was conducted in 2006 about the impact of HIV/AIDS among 116 affected and 109 control families by measuring the HRQL. Affected children's score was significantly lower on self report than children unaffected families. Caregivers of 
children from affected families reported lower scores than those from unaffected families. This study reported that children suffered from HIV had lower score on HRQL than those from unaffected families [6].

In South Africa $28.7 \%$ of the HIV infection total and one-third of infections among youth and children was reported in KwaZulu-Natal province. Significant relationships were observed between physical functioning and length of time on medications, comorbid health problems, and social support. The degree of prediction was analyzed by using a linear regression model. The predictor variables explained $29 \%$ of the variance in physical functioning. This shows that comorbid health problems were less among those individuals who reported a greater length of time on medications and better physical functioning was seen among those who had greater social support. [7].

A study of the quality of life (QOL) using Pediatric Quality of Life 4.0 (PedsQL) Inventory was studied among 97 residents including 52 boys and 45 girls at an institutional care home in Bangalore, India. Caregivers perceived children to have an overall higher QOL than was self-reported by children (total score 83 vs. 78). The findings indicated that self-reported QOL decreased with age of the child, while caregiver-reported QOL increased with age, suggesting a need to ensure greater psychological support for older children. Physical measures showed the children's clinical severity of disease remained well controlled living in this residential, values-based care home [8].

Relationship between perceived social support (PSS) and psychosocial well-being was studied among 1299 rural children suffering HIV/AIDS in central China. The findings of the study showed that PSS was highly correlated with psychosocial well-being of children. These correlations varied by functions and sources of the PSS as well as different psychosocial outcomes. There was a strong association between PSS and psychosocial outcomes among children affected by HIV/AIDS [9].

A cross sectional study was performed by Katherine in United States on HIV related quality of life was examined among 179 adolescent and young adult women. Depression, stigma, social support, and illness acceptance were the other variables studied. Significant association was found between depression $(p=.006)$, illness acceptance $(p<.001)$, social support $(p=.001)$, and current life satisfaction and depression $\quad(p=.012)$, illness acceptance $(p=.015)$, and illness burden current life satisfaction. HIV stigma with current life was found to be associated with illness related anxiety. [10].

This study aimed at comparing quality of life and social support of CLHIV who attended ART plus centre. This paper describes the quality of life of CLHIV in South India.

\section{Methods}

The study used survey approach with a descriptive correlational survey design. This study compared quality of life and social support of CLHIV. The subjects were 50 children living with HIV attended ART Plus centre of one of the districts of South India. Thus the subjects CLHIV selected using purposive sampling in the year 2013. CLHIV in age between 10-18 years, diagnosed positive for HIV/AIDS and are on anti-retroviral therapy attending ART Plus centre of District Hospital were included in the study. Children who were diagnosed positive for HIV/AIDS but not on ART and children with known or documented mental illnesses were excluded from the study.

The study protocol was approved by the Institutional Ethical Committee (IEC) of Kasturba Hospital Manipal, India. All the participants were informed of the purpose and procedures of the study, written informed consent from parents/care takers and assent from the child was obtained, and they were told that anonymity would be preserved.

\subsection{Data collection}

The data for this study was collected by the researcher among 50 children. Each child was interviewed by the researcher after completion of all formalities of ART plus center. Child along with caregivers were made to sit in a separate room to maintain confidentiality. Informed consent obtained from parents/care takers and assent from the child is obtained. Subject information was explained and assured that their responses will be kept confidential and will be only used for the study purpose. Confidentiality and anonymity of the participants were maintained throughout the study. Data pertaining to quality of life, social support was obtained from the children and background information was obtained from parents and children.

\subsection{Measures}

For assessing quality of life, standardized Horizon's questionnaire was used.. Highest score to each item was scored as five and lowest score to each item was one. Therefore the maximum possible score was 85 and the minimum score was 17 . To analyze and interpret the quality of life, the scale was categorized as poor 
and good quality of life. This questionnaire was translated into Kannada language. The content validity of the tool was established and reliability co-efficient of the quality of life scale was 0.747 .

For assessing social support, standardized Horizon's tool was used. This tool consists of 39 items. Out of which 4 items were open ended questions. Remaining 35 items were scored as 0 to 4 . Therefore the maximum score was 140 and the minimum score was 0 . To analyze and interpret the social support, the scale was categorized as high support and low support. This questionnaire was translated into Kannada language. The content validity of the tool was established and reliability co-efficient of the social support scale was 0.904 .

Both quality of life and social support questionnaire were pretested and piloted before the main study. The pilot study was conducted among 10 children during their visit to ART centre. The study was found to be feasible and hence no modifications were made in the main study.

\subsection{Data analysis}

Descriptive statistics were calculated for each variable. To find the association between quality of life, social support, with demographic variables, Chi-square test was used. Pearson's Correlation was performed for the quality of life and social support. SPSS 16.0 version was employed for all analyses.

\subsection{Demographic characteristics}

\section{Results}

Among 50 CLHIV, $46.1 \%$ of the subjects belonged to the age group of $16-18$ years; $56 \%$ of subjects were males, Majority $62 \%$ subjects' father's and $32 \%$ subjects' mother's cause of death was AIDS. Majority subjects' HIV was diagnosed between the age group of 1-6 years (22 out of 50 i.e 44\%) (TABLE1).

Table 1: Distribution of CLHIV based on demographic characteristics. $\mathbf{n = 5 0}$

\begin{tabular}{|c|c|c|c|}
\hline Sl. no & Demographic variables & Frequency & Percentage $(\%)$ \\
\hline \multirow[t]{4}{*}{1} & Age(in years) & & \\
\hline & $10-12$ & 7 & 14 \\
\hline & $13-15$ & 20 & 40 \\
\hline & $16-18$ & 23 & 46 \\
\hline \multirow[t]{3}{*}{2} & Gender & & \\
\hline & Male & 28 & 56 \\
\hline & Female & 22 & 44 \\
\hline \multirow[t]{3}{*}{3} & Father & & \\
\hline & Living & 11 & 22 \\
\hline & Dead & 39 & 78 \\
\hline \multirow[t]{4}{*}{4} & Age of HIV diagnosed in years & & \\
\hline & $1-6$ & 22 & 44 \\
\hline & $7-12$ & 19 & 38 \\
\hline & $13-18$ & 9 & 18 \\
\hline
\end{tabular}

\subsection{Quality of life and social support}

Frequency and percentage distribution of quality of life and social support showed that $60 \%$ of the subjects were found to be poor quality of life and (86\%) of the subjects were found to have low social support (TABLE 2,3).

Table 2: Frequency and Percentage distribution Mean, Median, Standard Deviation of QOL of CLHIV $\mathbf{n}=\mathbf{5 0}$

\begin{tabular}{lccccc}
\hline QOL & Frequency & Percentage & Mean & Median & $\begin{array}{c}\text { Standard } \\
\text { deviation }\end{array}$ \\
\hline $\begin{array}{l}\text { Good } \\
(52-85)\end{array}$ & 20 & 40 & & & \\
$\begin{array}{l}\text { Poor } \\
(17-51)\end{array}$ & $\mathbf{3 0}$ & $\mathbf{6 0}$ & 50.66 & 50.0 & \pm 6.213 \\
\hline
\end{tabular}

Table 3: Frequency, percentage, mean, median and standard deviation of Social support of CLHIV

\begin{tabular}{|c|c|c|c|c|c|}
\hline \multicolumn{6}{|c|}{$n=50$} \\
\hline Social support & Frequency & Percentage & Mean & Median & $\begin{array}{l}\text { Standard } \\
\text { deviation }\end{array}$ \\
\hline $\begin{array}{l}\text { High support } \\
(0-70)\end{array}$ & 7 & 14 & & & \\
\hline $\begin{array}{l}\text { Low support } \\
\text { (71-140) }\end{array}$ & 43 & 86 & 83.16 & 84 & \pm 9.717 \\
\hline
\end{tabular}


3.3. Relationship between quality of life and social support of CLHIV

Table 4: Relationship between quality of life and social support of CLHIV n=50

\begin{tabular}{lcc}
\hline Variables & Correlation coefficient & $p$-value \\
\hline Quality of life & 0.293 & 0.039 \\
Social support & & \\
\hline
\end{tabular}

Pearson's Correlation performed for the quality of life and social support showed a statistically significant weak positive relationship between quality of life and social support $(\mathrm{r}=.293 ; p=.039)$ TABLE 4

\subsection{Association between quality of life, social support and demographic variable.}

Chi square test was performed to find the association between quality of life, social support and demographic variable. The results showed a significant association between quality of life, father living/dead $(p=0.018)$. There was a significant association found between, father living/dead $(\boldsymbol{p}=0.018)$ with quality of life at 0.05 level. Whereas no association was observed between variables includes age, gender, both parent's status includes parents living or dead.

\section{Discussion}

The main findings of the study were that the CLHIV were found to have poor quality of life and low social support. Results of this study is similar to the study carried out in four townships of Longchuan County in Yunnan[6] and study of caregivers of HIV infected children in Thailand [5]

This study is conducted among is limited to children who are attending to ART Plus centre of one of the Districts. Studying all children who were diagnosed to have HIV irrespective of ART treatment may give more information about their quality of life and social support.

\section{Conclusion}

We performed this study to find the correlation between quality of life and social support. We found that majority of the CLHIV had poor quality of life and low social support. To improve and maintain quality of life and social support among CLHIV, it is therefore important to conduct intervention that will empower CLHIV and help them to live in the society. Intervention studies are required to confirm these findings.

\section{Acknowledgements}

We would like to thank all the CLHIV who took part in this study.

\section{References}

[1]. UNAIDS. unaids.org. [Online].; 2013 [cited 2014 September 8. Available from: HYPERLINK "http://www.unaids.org/en/media/unaids/contentassets/documents/epidemiology/2013/gr2013/UNAIDS_Global_Report_2013_en.p df"

[2]. UNAIDS. People Living with HIV Stigma Index- Asia Pacific Regional Analysis. ; 2011.

[3]. NACO. Annual Report. ; 2012-2013.

[4]. Gupta M, Nanda S, Kaushik JS. Quality of Life in Symptomatic HIV Infected Children. Indian Pediatrics. 2013;: p. 1145-1147.

[5]. Oberdorfer, Louthrenoo, Puthanakit, Sirisanthana, Sirisanthana T. Quality of Life Among HIV-Infected Children in Thailand. Journal of the International Association of Physicians in AIDS Care. $2008 ;:$ p. 141-147.

[6]. Tao Xu. Quality of life of children living in HIV/AIDS-affected families in rural areas in Yunnan, China. AIDS Care. 2010;: p. 390396.

[7]. Busisiwe P. Ncama, Dean Wantland, Busisiwe R. Bhengu, Chris McGibbon, Sheila M. Davis, Inge B. Corless and Patrice K. Nicholas. Quality of life and physical functioning in HIV-infected individuals receiving antiretroviral therapy in KwaZulu-Natal, South Africa. Nursing \& Health Sciences. 2008; 10( 4): p. 266-272.

[8]. Quality of life and psychosocial well-being among children living with HIV at a care home in Southern India. Vulnerable Children and Youth Studies: An International Interdisciplinary Journal for Research, Policy and Care. 2014; 9(4): p. 345-352.

[9]. Zhao G, Li X, Fang X, Zhao J, Hong Y, Lin X, Stanton B. Functions and sources of perceived social support among children affected by HIV/AIDS in China. AIDS care. 2011;: p. 671-9.

[10]. Katherine Andrinopoulos GC. Health related Quality of Life and Psychosocial correlates amon HIV-infected Adolescents and Young Adult Women in the US. AIDS Educ Prev. 2011;: p. 367-381.

[11]. Mohit Gupta SNJSK. Quality of Life in Symptomatic HIV Infected Children. Indian Pediatrics. 2013; 50(15): p. 1145-1147. 